\title{
大豆根部水压胁迫耐逆指数遗传体系解析
}

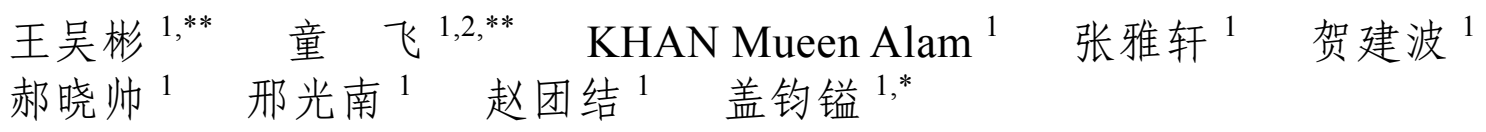

1 南京农业大学大豆研究所 / 国家大豆改良中心 / 农业农村部大豆生物学与遗传育种重点实验室(综合) / 作物遗传与种质创新国家 重点实验室 / 江苏作物生产协同创新中心, 江苏南京 $210095 ;{ }^{2}$ 江苏徐淮地区徐州农业科学研究所, 江苏徐州 221131

摘 要: 大豆是重要的植物蛋白质和植物油脂来源, 干旱是影响大豆产量的重要环境因子之一。为解析大豆耐旱性 的遗传基础, 本研究在 PEG 水压胁迫条件下, 对由 409 个家系组成的巢式关联作图群体(具有 1 个共同亲本的 2 个重 组自交系群体组成)进行叶片脯氨酸含量测定, 通过限制性二阶段多位点全基因组关联分析(restrictive two-stage multilocus genome-wide association study, RTM-GWAS), 解析了大豆根部水压胁迫耐逆指数(root hydraulic stress tolerance index, RHSTI)的遗传体系。结果表明, 在春季和夏季环境下, 3 个亲本蒙 8260 (共同亲本)、通山薄皮黄豆甲和正阳白 毛平顶在 RHSTI 上均存在显著差异, 其衍生群体 RHSTI 表型变异丰富, 变幅分别为 $0.11 \sim 2.94$ 和 $0.03 \sim 1.93$, 遗传力 分别为 $97.7 \%$ 和 $97.9 \% ; 2$ 个环境联合分析发现, 家系遗传力和家系与环境互作遗传力分别为 $37.9 \%$ 和 $60.1 \%$, 说明群 体 RHSTI 的变异受遗传和环境共同控制。通过 RTM-GWAS 方法, 共检测到 45 个与 RHSTI 相关的 QTL, 分布在大 豆 18 条染色体上, 可以解释 $37.58 \%$ 的表型变异, 其中 7 个 QTL 的表型贡献率超过 $1 \%$, 为大贡献位点; 这些 QTL 中, 有 34 个位点与环境存在显著互作, 可以解释 $12.50 \%$ 的表型变异。结合 PEG 胁迫下大豆转录组数据, 在定位区间 500 $\mathrm{kb}$ 范围内共筛选到 38 个差异表达基因, 可归为 $\mathrm{ABA}$ 响应因子、逆境响应因子、转录因子、发育因子、蛋白代谢因 子、未知功能和其他等 7 类, 其中逆境响应因子、转录因子和发育因子是最大的 3 类; 其中位于主效位点的 6 个基 因, 与 $\mathrm{ABA}$ 响应因子、逆境响应因子、转录因子相关, 应为主要候选基因。上述结果表明, 大豆耐旱性是一个由 多位点、多基因控制的复杂数量性状, 且与环境存在互作, 遗传基础复杂。研究结果为大豆耐旱性分子育种提供 了依据。

关键词：大豆; 根部水压胁迫耐逆指数; 脯氨酸含量; 数量性状位点; 巢式关联作图群体

\section{Detecting QTL system of root hydraulic stress tolerance index at seedling stage in soybean}

WANG Wu-Bin ${ }^{1, * *}$, TONG Fei ${ }^{1,2, * *}$, KHAN Mueen-Alam ${ }^{1}$, ZHANG Ya-Xuan ${ }^{1}$, HE Jian-Bo ${ }^{1}$, HAO Xiao-Shuai ${ }^{1}$, XING Guang-Nan ${ }^{1}$, ZHAO Tuan-Jie ${ }^{1}$, and GAI Jun-Yi ${ }^{1, *}$

\footnotetext{
${ }^{1}$ Soybean Research Institute, Nanjing Agricultural University / National Center for Soybean Improvement / MOA Key Laboratory for Biology and Genetic Improvement of Soybean (General), Ministry of Agriculture and Rural Affairs / National Key Laboratory of Crop Genetics and Germplasm Enhancement / Jiangsu Collaborative Innovation Center for Modern Crop Production, Nanjing 210095, Jiangsu, China; ${ }^{2}$ Xuzhou Institute of Agricultural Sciences of Xuhuai Region of Jiangsu, Xuzhou 221131, Jiangsu, China
}

本研究由中央高校基本科研业务费专项(KYZZ201901), 国家自然科学基金项目(31601325), 教育部长江学者和创新团队项目 (PCSIRT_17R55)，国家现代农业产业技术体系建设专项(CARS-04)和江苏省 JCIC-MCP 项目资助。

This study was supported by the Fundamental Research Funds for the Central Universities (KYZZ201901), the National Natural Science Foundation of China (31601325), the MOE Program for Changjiang Scholars and Innovative Research Team in University (PCSIRT_17R55), the China Agriculture Research System (CARS-04), and the Jiangsu JCIC-MCP.

*通信作者(Corresponding author): 盖钧镒, E-mail: sri@njau.edu.cn

** 同等贡献( Contributed equally to this work)

第一作者联系方式: 王吴彬, E-mail: soybeanwang@163.com; 童飞, E-mail: ttongfei@126.com

Received (收稿日期): 2020-07-31; Accepted (接受日期): 2020-11-13; Published online (网络出版日期): 2020-12-23.

URL: https://kns.cnki.net/kcms/detail/11.1809.S.20201222.1802.009.html 


\begin{abstract}
Soybean is an important source of plant protein and vegetable oil in the world. Drought is one of the important environmental stress factors affecting soybean yield. To explore the genetic base of drought tolerance in soybean, a nested association mapping population composed of two sets of recombinant inbred lines with a common parent in a total of 429 lines was investigated for leaf proline content under PEG simulated drought stress. The genetic system of root hydraulic stress tolerance index (RHSTI) was analyzed using the RTM-GWAS (restrictive two-stage multilocus genome-wide association study). The results showed that there were significant differences in RHSTI among the three parents under two different environments in spring and summer, and among the nested association mapping population with the variation range of $0.11-2.94$ and $0.03-1.93$, respectively. The heritability values of Line and Line $\times$ Environment were $37.9 \%$ and $60.1 \%$, respectively, indicating that the variation of RHSTI was greatly affected by the environment. Using the RHSTI data and 6137 SNPLDB markers, a total of 45 main effect QTLs were detected on 18 chromosomes, which could explain a total of $37.58 \%$ phenotypic variation, including 7 large contribution QTLs with $R^{2}$ more than $1 \%$. Among them, 34 QTLs with QTL $\times$ Environment effect explained $12.50 \%$ of the phenotypic variation. Combined with the transcriptome data under PEG stress, totally 38 differentially expressed genes were identified within a QTL \pm $500 \mathrm{~kb}$, which can be grouped into different biological categories, including ABA responders, stress responders, transcription factors, development factors, protein metabolism factors, unknown functions and others, with stress responses, transcription factors and development factors as the major parts. In summary, the results indicated that the drought tolerance of soybean was a complex quantitative trait, with the complex genetic basis controlling by multiple loci, multiple genes and interaction with the environment. The present results can lay the foundation of molecular breeding for drought tolerance in soybean.
\end{abstract}

Keywords: soybean; root hydraulic stress tolerance index; proline content; QTL; nested association mapping population

大豆[Glycine max (L.) Merr.]是重要的植物蛋白 质和油料作物, 近年来在世界上迅速发展。地球上 $41 \%$ 的陆地面积属于干旱地区 ${ }^{[1]}$, 即使湿润与半湿 润地区，由于气候变化问题，季节性干旱也经常发 生 ${ }^{[2]}$ 。干旱或持续缺水是限制大豆生长发育及产量 实现的最重要环境因素。温室及田间研究表明，水 分胁迫能使大豆产量降低 $24 \% \sim 50 \%{ }^{[3-4]}$, 严重情况 下甚至绝收。因而, 解析大豆耐旱性的遗传机制, 具 有重要的育种价值。

干旱可发生在大豆生长发育的不同阶段，包括 苗期、开花期和鼓粒期等。苗期耐旱性鉴定具有试验 周期短的特点, 能有效加快耐旱性遗传研究及育种利 用进程。耐旱性评价方法有多种，包括田间鉴定法 ${ }^{[5]}$ 、 干旱棚盆栽法 ${ }^{[6]}$ 、实验室法 ${ }^{[7]}$ (水培法和高渗溶液法 等)等。耐旱性评价指标包括株高、干重等生长性状 和叶片相对含水量和脯氨酸含量等生理生化性状等。

在水分胁迫下，植物适应逆境，主要通过积累大 量有机或无机物质来调节细胞质的渗透势，保护酶、 蛋白质和生物膜系统 ${ }^{[8]}$ 。有机物质以脯氨酸、可溶性 糖和甜菜碱甘露醇为主, 其中, 脯氨酸是一种溶解度 高的小分子渗透物质，具有良好的水合性，在植物受 到逆境环境时能够大量积累，可有效减少植物体内 水分散失，实现细胞和组织的保水或吸水，抑制逆境 对植物细胞膜透性的破坏，同时提高抗氧化酶的活 性, 从而减轻逆境造成的伤害 ${ }^{[9]}$ 。目前, 脯氨酸含量 是植物抗逆生理生化研究中通用的一项重要指标 ${ }^{[10]}$ 。

由于大豆耐旱性易受环境影响，表型鉴定困难，导 致了其遗传研究滞后于其他性状。截止目前, SoyBase 网 址(https://www.soybase.org/)共收录 88 个耐旱性 QTL, 其 中 62 个 QTL 来自于 9 个不同杂交组合衍生的重组自交 系群体, 另外 26 个 QTL 是通过不同群体 QTL 元分析获 得, 这些 QTL 分布在除 15 号染色体以外的所有染色体 上, 其中 12 号和 17 号染色体上分布的 QTL 最多, 可能 携带主效耐旱性位点, 具体信息见表 1 。

为解析大豆苗期耐旱性的遗传基础, 前期研究 构建了一套来自蒙 8206 (Meng 8202, M) 、通山薄皮黄 豆甲 (Tongshanbopihuangdoujia，T) 和正阳白毛平顶 (Zhengyangbaimaopingding, Z)的巢式关联作图群体 (population from Meng 8202, Tongshanbopihuangdoujia and Zhengyangbaimaopingding, MTZ), 并以根长、茎 长、植株长度 (根+茎)和干重等生长性状为指标, 检 测到 157 个耐旱性 QTL ${ }^{[19-20]}$ 。在此基础上, 本研究 将耐旱指标拓展到生理性状, 在 $15 \%$ PEG-6000 模 拟干旱条件下, 以脯氨酸含量为基础的根部水压胁 迫耐逆指数为指标, 分别在春季和夏季 2 个环境下 评价 MTZ 群体苗期耐旱性; 然后, 联合 MTZ 群体 表型与基因型, 利用限制性二阶段多位点全基因组 关联分析方法(restricted two-stage multilocus genomewide GWAS, RTM-GWAS)，解析了 MTZ 群体苗期根 部水压胁迫耐逆指数的遗传体系，以期发掘控制大 豆苗期耐旱性的主要位点及其候选基因，为大豆耐 旱性分子育种提供依据。

\section{1 材料与方法}

\section{1 试验材料}

为解析大豆耐旱性的遗传基础, 课题组前期构 建了一套以敏旱材料蒙 $8206(\mathrm{M}$, 熟期组 V)为共同 
表 1 文献报道的部分大豆耐旱性位点信息

Table 1 Information of drought tolerance QTLs reported in the literatures

\begin{tabular}{|c|c|c|c|c|}
\hline 作图群体 & 评价指标 & QTL 数目 & 染色体 $(\mathrm{QTL} \text { 数目 })^{\mathrm{a}}$ & 参考文献 \\
\hline Mapping population & & & Chromosome (QTL number) & Reference \\
\hline Benning/PI 416937 & 萎菣指数 Canopy width & 7 & 2(1) 4(1) 5(1) 12(1) 14(1) 17(1) 19(1) & [11] \\
\hline KS4895/Jackson & 萎菣指数 Canopy width & 4 & $8(1) 13(1) 14(1) 17(1)$ & {$[12]$} \\
\hline 93705KS4895/Jackson & 萎菣指数 Canopy width & 13 & $2(3) 4(1) 5(2) 6(2) 8(1) 14(1) 17(3)$ & [13] \\
\hline 08705KS4895/Jackson & 萎菣指数 Canopy width & 7 & $9(1) 11(2) 12(1) 16(1) 17(2)$ & [13] \\
\hline KS4895/ PI 424140 & 萎菣指数 Canopy width & 4 & $11(1) 17(1) 19(2)$ & [13] \\
\hline Benning/PI 416937 & 萎菣指数 Canopy width & 4 & $2(3) 19(1)$ & [13] \\
\hline NN1138-2/Kefeng 1 & 耐旱系数 Drought index & 10 & $1(1) 5(2) 6(1) 7(1) 12(1) 16(1) 17(2) 20(1)$ & {$[14]$} \\
\hline Benning/PI 416937 & 叶片电导率 Hydraulic conductance & 4 & $3(1) 5(1) 10(1) 12(1)$ & {$[15]$} \\
\hline S-100/Tokyo & 水分利用率 Water use efficiency & 3 & $4(2) 19(1)$ & {$[16]$} \\
\hline Young/PI 416937 & 水分利用率 Water use efficiency & 6 & $12(2) 16(3) 18(1)$ & {$[17]$} \\
\hline QTL 元分析 Meta-QTL & & 26 & $2(10) 5(3) 11(4) 12(2) 17(6) 19(3)$ & {$[18]$} \\
\hline
\end{tabular}

a: 括号外数字代表染色体号, 括号内数字代表 QTL 数目。

a: the number outside the parentheses is the code of chromosome, and the number in the parentheses is the number of QTLs.

母本，耐旱材料通山薄皮黄豆甲 ( T, 熟期组 VI) 和正 阳白毛平顶 $(Z$ ，熟期组 V)为父本的巢式关联作图群 体 MTZ。MTZ 群体共包含 409 个重组自交系，其中 285 份家系来自蒙 $8206 \times$ 通山薄皮黄豆甲(M8206 $\times$ Tongshanbopihuangdoujia, MT), 124 份来自蒙 $8206 \times$ 正阳白毛平顶(M8206 × Zhengyangbaimaopingding, MZ)。MT 和 MZ 群体构建方法如下: 以蒙 8206 为 共同母本, 分别与通山薄皮黄豆甲和正阳白毛平顶 杂交, 获得 $F_{1}$, 后经单籽传法自交至 7 代, 得到重组 自交系群体。上述材料均由南京农业大学国家大豆 改良中心种质资源库提供。

\section{2 试验设计及群体表型评价}

试验在南京农业大学国家大豆改良中心温室中 进行，采用完全随机区组设计，3 次重复，分别在春 季(2017 年 4 月, 温度大约 $6 \sim 24^{\circ} \mathrm{C}$ )和夏季(2017 年 6 月, 温度大约 $\left.21 \sim 32^{\circ} \mathrm{C}\right) 2$ 个环境下进行。鉴定方法 如下: 首先, 剪裁边长约 $20 \mathrm{~cm}$ 的正方形滤纸, 选取 MTZ 群体及其 3 个亲本各 7 粒种子, 均匀排列在滤 纸上方三分之一处，匀速卷动滤纸，保证种子不移 位，卷好后分上中下 3 处将纸卷用胶带固定，然后 垂直插入 $500 \mathrm{~mL}$ (直径 $9 \mathrm{~cm}$, 高 $11 \mathrm{~cm}$ )的塑料杯中, 杯中注水; 选择长势一致的大豆植株，每个处理留 2 株，为保证不伤及试验所用幼苗，将其余幼苗用剪 刀剪除; 待植株生长至 V1 期(第 1 片三出复叶展开) 时，对幼苗进行含有 15\% PEG-6000 (聚乙二醇)的 Hoagland 营养液胁迫处理，营养液每周更换 1 次; 待植株长到 V2 期(第 2 片三出复叶展开)时, 采取
第 2 片三出复叶中间小叶进行叶片脯氨酸含量测 定。

采用酸性茚三酮显色法测定叶片脯氨酸含 量 ${ }^{[21-22]}$, 根据耐旱亲本通山薄皮黄豆甲和正阳白毛 平顶叶片脯氨酸含量均值计算每份材料的根部水压 胁迫耐逆指数(RHSTI), 以增加不同重复和环境间 表型数据的可比性，计算公式为 RHSTI $_{i j k}=$ $\mathrm{LPC}_{i j k} /\left(\mathrm{LPC}_{i j T}+\mathrm{LPC}_{i j \mathrm{z}}\right) / 2, \mathrm{RHSTI} \mathrm{i}_{i j k}$ 代表第 $i$ 个环境、 第 $j$ 次重复中第 $k$ 个家系的根部水压胁迫耐逆指数, $\mathrm{LPC}_{i j k}$ 表示第 $i$ 个环境、第 $j$ 次重复中第 $k$ 个家系的 叶片脯氨酸含量; $\mathrm{LPC}_{i j T}$ 表示第 $i$ 个环境、第 $j$ 次重 复中耐旱亲本通山薄皮黄豆甲的叶片脯氨酸含量; $\mathrm{LPC}_{i j T}$ 表示第 $i$ 个环境、第 $j$ 次重复中耐旱亲本正阳 白毛平顶的叶片脯氨酸含量。RHSTI 值越大，表示 材料越耐旱。

\subsection{MTZ 群体基因型鉴定}

采用 CTAB 法从大豆叶片中提取大豆基因组 DNA, 经 Taq I 酶切后, 选取 400 700 bp 之间的 DNA 片段，利用 Illumina HiSeq 2000 测序仪的多元 乌枪法基因分型策略 ${ }^{[23]}$, 测定 DNA 片段两端碱基 序列, 可读取长度 $90 \mathrm{bp}$ 。采用 SOAP2 软件 ${ }^{[24]}$ 将测 序获得的原始测序 reads 与 Williams 82 参考基因组 (Glyma. Wm82.a1.v 1.1)进行比对。利用基于贝叶斯 模型的 realSFS 软件 ${ }^{[25]}$ 进行 SNP 基因型的鉴定。用 以下标准对 MTZ群体的 SNP进行篮选, 缺失和杂合 率 $\leq 30 \%$ ，最小等位基因频率 $\geq 1 \%$ （如果存在第 3 个或更多个等位基因时，则用缺失等位基因替换)。 
通过 RAD-seq 测序, MZ 和 MT 群体最终分别获得 66,677 和 55,958 个 SNP, 用于后续划分 SNP 连锁不 平衡区段 (SNP linkage disequilibrium block, SNPLDB)标记。此部分工作由深圳华大基因科技有 限公司完成。

对 MTZ群体 SNP 数据进行严格过滤，仅保留基 因分型率 $80 \%$ 以上的 SNP 标记, 通过并集方式，把 MTZ 群体的 SNP 数据进行合并, 获得 55,936 个 SNP。使用 fastPHASE 软件 ${ }^{[26]}$ 对缺失 SNP 进行填补, 利用 Haploview 软件 ${ }^{[27]}$ 根据 MTZ 群体的 55,936 个 SNP 标记划分单倍型区段, 使用默认设置的置信区 间法定义区段, 最大距离和最小 MAF 分别设置为 $200 \mathrm{~kb}$ 和 0.01 。位于 1 个 LD (linkage disequilibrium) 区段内紧密连锁的多个 SNP, 整合在一起作为该基 因组区域的一段序列, 划分为 1 个 SNPLDB 标记。 基于以上方法, MTZ 群体共检出 55,936 个 SNP 组成 的 6137 个 SNPLDB 标记用于后续分析。该群体基 因型已应用于以根长、茎长、植株长度 $($ 根 + 茎)和干 重等生长性状为指标的耐旱性遗传研究 ${ }^{[19-20]}$ 。

\subsection{MTZ 群体全基因组关联分析}

采用贺建波等 ${ }^{[28]}$ 提出的限制性二阶段多位点全 基因组关联分析方法(RTM-GWAS)进行大豆根部水 压胁迫耐逆指数遗传解析。所用分子标记是 MTZ 群 体的 6137 SNPLDB，表型数据是 2 个环境下大豆根 部水压胁迫耐逆指数(含每个环境下的 3 次重复数 据)。RTM-GWAS 关联分析分 2 个阶段进行，在第 1 阶段，基于简单线性模型(simple linear model)进行 单位点关联检验 (single-locus association test), 对 SNPLDB 标记进行初步篮选(从 6137 个 SNPLDB 中 选出 2708 个); 在第 2 阶段, 对第 1 阶段篮选到的 2708 个显著标记, 利用多位点逐步回归模型进行䇻 选，并估计等位变异的效应。在这 2 个阶段中，将基 于 SNPLDB 标记的个体间遗传相似系数矩阵的前 10
个特征向量作为协变量进行群体结构控制。为检测 到更多的候选关联位点, 初步篮选标记和多位点逐 步回归关联分析时, 显著水平分别为 $P=0.05$ 和 $P=0.01$ ，遗传力控制在 $37.9 \%$ 以内。

\subsection{MTZ 群体 QTL-等位变异矩阵构建}

将 RTM-GWAS 关联分析检测到的 QTL 及等位 变异效应构建 QTL-等位变异(QTL-allele)矩阵, 构 建方法如下：以 409 个家系为横坐标，以关联 SNPLDB 位点为纵坐标, 每个单元格表示某一家系 在特定关联标记上的等位变异效应。为简化表示该 矩阵, 用不同颜色梯度来表示等位变异效应值的变 化趋势, 红色表示增效等位变异, 逐步过渡到蓝色 来表示减效等位变异。

\section{6 大豆苗期耐旱性候选基因分析}

以课题组前期鉴定的耐旱材料光泽黄荚豆为材 料，用 Hoagland 营养液培养至 V1 期，后用含有 $15 \%$ PEG-6000 的 Hoagland 营养液进行胁迫处理 $12 \mathrm{~h}$, 分 别取处理前后的叶片进行转录组测序, 测序深度为 5 倍。该测序工作委托上海伯豪生物技术有限公司 进行。联合关联分析和转录组测序结果, 将位于 $\mathrm{QTL} \pm 500 \mathrm{~kb}$ 区间内(衰减距离)的差异表达基因认为 本研究检测到的耐旱性候选基因。

\section{2 结果与分析}

\section{1 大豆 MTZ 群体及其亲本根部水压胁迫耐逆} 指数表现

在春季和夏季 2 个环境中, 3 个亲本蒙 8206 、正 阳白毛平顶和通山薄皮黄豆甲的根部水压胁迫耐逆 指数 RHSTI 分别为 0.35 与 $0.17 、 1.05$ 与 1.04 和 0.95 与 0.96 , 共同亲本蒙 8206 较其他 2 个亲本 RHSTI 存在显著差异, 且较低, 说明其耐旱性较弱(表 2 和 图 1)。在春、夏 2 个环境下, MTZ 群体 RHSTI 均表 现连续分布，且具有较高遗传力，分别为 $97.2 \%$ 和

表 2 MTZ 群体及其亲本根部水压胁迫耐逆指数 RHSTI 的描述性统计

Table 2 Descriptive statistics of root hydraulic stress tolerance index (RHSTI) in the MTZ population

\begin{tabular}{|c|c|c|c|c|c|c|c|c|c|c|c|c|c|c|c|c|c|}
\hline \multirow{3}{*}{$\begin{array}{c}\text { 环境 } \\
\text { Environment }\end{array}$} & \multirow{3}{*}{ M } & \multirow{3}{*}{ Z } & \multirow{3}{*}{$\mathrm{T}$} & \multicolumn{14}{|c|}{ MTZ } \\
\hline & & & & \multicolumn{10}{|c|}{ 组中值(频数) Value of class mid-point (frequency) } & \multirow{2}{*}{$\begin{array}{c}\text { 变幅 } \\
\text { Range }\end{array}$} & \multirow{2}{*}{$\begin{array}{l}\text { 平均数 } \\
\text { Mean }\end{array}$} & \multirow{2}{*}{$\begin{array}{c}\text { 变异系数 } \\
\text { CV }(\%)\end{array}$} & \multirow{2}{*}{$\begin{array}{c}\text { 遗传力 } \\
h^{2}\end{array}$} \\
\hline & & & & 0.1 & 0.3 & 0.5 & 0.7 & 0.9 & 1.1 & 1.3 & 1.5 & 1.7 & $>1.9$ & & & & \\
\hline 春季 Spring & 0.35 & 1.05 & 0.95 & 1 & 24 & 159 & 128 & 57 & 15 & 9 & 2 & 1 & 9 & $0.11-2.94$ & 0.69 & 13.2 & 97.2 \\
\hline 夏季 Summer & 0.17 & 1.04 & 0.96 & 38 & 56 & 75 & 100 & 61 & 34 & 20 & 5 & 0 & 2 & $0.03-1.93$ & 0.65 & 12.1 & 97.9 \\
\hline 平均值 Mean & 0.26 & 1.05 & 0.95 & 2 & 41 & 115 & 159 & 55 & 19 & 5 & 6 & 0 & 5 & $0.09-2.80$ & 0.68 & 12.7 & 37.9 \\
\hline
\end{tabular}

M: 蒙 8206; Z: 正阳白毛平顶; T: 通山薄皮黄豆甲; MTZ: 3 个亲本衍生的巢式关联作图群体。

M, Z, T, and MTZ represent M8206, Zhengyangbaimaopingding, Tongshanbopihuangdoujia, and their derived population, respectively. 


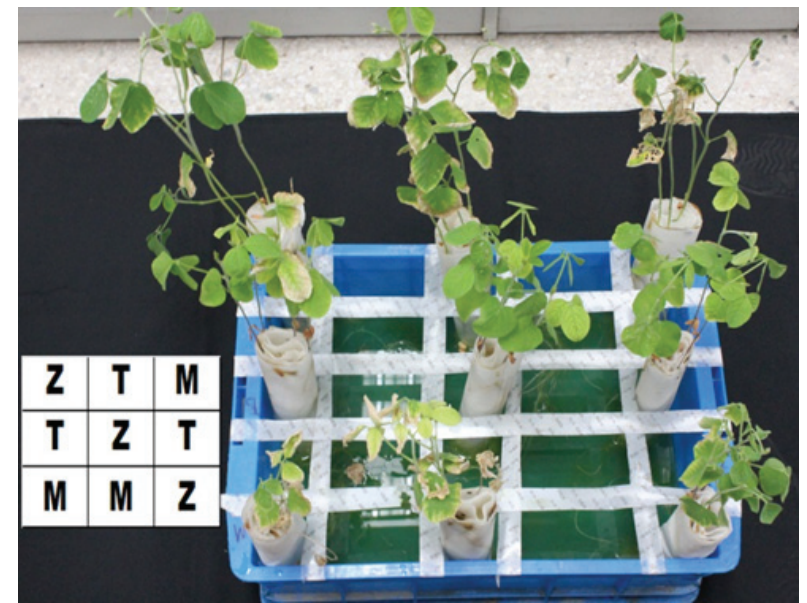

图 1 PEG 模拟干旱条件下 3 个亲本耐旱性表现

Fig. 1 Drought performance of three parents under PEG simulated stress

图片左侧的字母代表亲本在右侧蓝色箱子中的位置。缩写同表 2。 The letters on the left in the figure represent the positions of three parents in the blue box on the right. Abbreviations are the same as those given in Table 2 .

97.9\%，表明 PEG 模拟干旱条件下大豆 RHSTI 属于 多基因控制的数量性状。在春季环境下, MTZ 群体 RHSTI 平均值为 0.69 , 变幅为 $0.11 \sim 2.94$; 在夏季环 境下, RHSTI 均值为 0.65 , 变幅为 $0.03 \sim 1.93$, 表明 MTZ 群体 RHSTI 存在广泛变异, 适合大豆耐旱性遗 传解析。在 2 个不同环境下, 误差变异系数分别为 $13.2 \%$ 和 $12.1 \%$ ，均低于 $15.0 \%$ ，说明本研究误差控 制较好。

对 2 个不同环境下 MTZ 群体的 RHSTI 进行联 合分析发现，群体在 RHSTI 上表现连续分布，均值 为 0.68 , 变幅为 $0.09 \sim 2.80$, 同样显示了广泛的表型 变异。联合方差分析结果显示，家系以及家系×环境 间均存在极显著差异。在 RHSTI 上, 家系遗传力为 $37.9 \%$ ，家系与环境互作遗传力为 $60.1 \%$, 这与叶片 脯氨酸含量在春季与夏季间差异较大相符，可能与 不同环境下气温差异较大有关(表 3)。

2.2 MTZ 群体根部水压胁迫耐逆指数 QTL-等 位变异(QTL-allele)体系解析

联合 MTZ 群体 2 个环境下的 RHSTI 表型数据 和 6137 个 SNPLDB 标记基因型数据, 通过 RTM-GWAS 方法，在遗传力控制在 37.9\%以内的情 况下, 共检测到了 45 个与根部水压胁迫耐逆指数显 著相关的 QTL, 显著水平 $(-\lg P)$ 变幅为 $11.7 \sim 73.0$, 12 号染色体上的位点 RHSTI12.2 显著性最高(图 2-A, $\mathrm{B})$; 这些位点中, 有 34 个位点与环境存在显著互作 (QEI, QTL × Environment interaction)，具体结果见
表 3 MTZ 群体根部水压胁迫耐逆指数 RHSTI 两环境联合方差 分析

Table 3 Joint analysis of variance of root hydraulic stress tolerance index (RHSTI) under two environments in the MTZ population

\begin{tabular}{lc}
\hline \multicolumn{1}{c}{$\begin{array}{c}\text { 变异来源 } \\
\text { Source of variation }\end{array}$} & $\begin{array}{c}F \text { 值 } \\
F \text {-value }\end{array}$ \\
\hline 家系 Line & $50.54^{* *}$ \\
环境 Environment & 0.80 \\
区组(环境) Block (Environment) & $94.89^{* *}$ \\
家系×环境 Line $\times$ Environment & $30.38^{* *}$ \\
\hline${ }^{* *}:$ 显著水平 $P=0.01$ 家系为固定效应; 环境、区组(环境)和家 \\
系×环境为随机效应。 \\
${ }^{* *}:$ significance at $P=0.01$. Line as a fixed effect; Environment, \\
Block (Environment) and Line $\times$ Environment as random effects.
\end{tabular}

表 4。检测到的 QTL 分布在大豆 Gm01、Gm02、 Gm03、Gm04、Gm06、Gm07、Gm08、Gm09、Gm10、 Gm11、Gm12、Gm13、Gm14、Gm15、Gm17、Gm18、 $\mathrm{Gm} 19$ 和 $\mathrm{Gm} 20$ 等 18 条染色体上, 每条染色上携带 有 1 个 $(\mathrm{Gm} 01 、 \mathrm{Gm} 04 、 \mathrm{Gm} 08$ 和 $\mathrm{Gm} 20)$ 至 4 个 $(\mathrm{Gm} 02$ 、 Gm09、Gm10 和 Gm19) QTL。这些位点总计解释 $37.58 \%$ 的表型变异，单个位点的贡献率变幅为 $0.46 \% \sim 3.09 \%$; 其中有 7 个 QTL 的贡献率 $\geq 1 \%$, 归 为大贡献 QTL，应为控制大豆耐旱性的主要位点, 累计解释 $12.00 \%$ 的表型变异; 另外 38 个 QTL 的表 型贡献率 $<1 \%$ ，归为小贡献 QTL，累计解释 $25.58 \%$ 的表型变异。这些 QTL 中, 有 33 个位点与环境存在 显著互作 $(\mathrm{QEI}$ 位点)，单个 $\mathrm{QEI}$ 的表型贡献率变幅为 0.06 1.70, 互作累计可以解释 $12.50 \%$ 的表型变异; 其中 3 个为大贡献位点, 累积贡献率 $4.35 \%, 30$ 个为 小贡献位点，累积贡献率 $8.15 \%$ 。根据遗传解析结果， RHSTI的遗传体系可以分解为 3 部分, 包括: 45 个主 效 QTL，可以解释 37.58\%的表型变异; 33 个 QTL 与 环境互作效应, 可以解释 $12.50 \%$ 的表型变异; 另外 $37.9 \%\left(h^{2}\right)+60.11 \%$ (互作遗传力) $-37.58 \%-12.50 \%=$ 47.93\%的表型变异, 可能是由未被检测到的微效 QTL 控制。鉴于本研究检测到的 QTL 与环境互作效 应可能由于春季和夏季不同光温条件引起的, 差异 条件较难衡量和重复, 且互作位点包含于主效位点 内, 所以后续的 QTL-等位变异矩阵和候选基因分析 依据主效位点进行。

2.3 MTZ 群体根部水压胁迫耐逆指数 QTL-allele 矩阵及三亲本后代育种潜力

在 45 个根部水压胁迫耐逆指数 QTL 上, MTZ 群体总共携带 123 个等位变异, 平均每个位点携带 2.73 个等位变异, 变幅为 $2 \sim 3$ 个。在 123 个等位变 


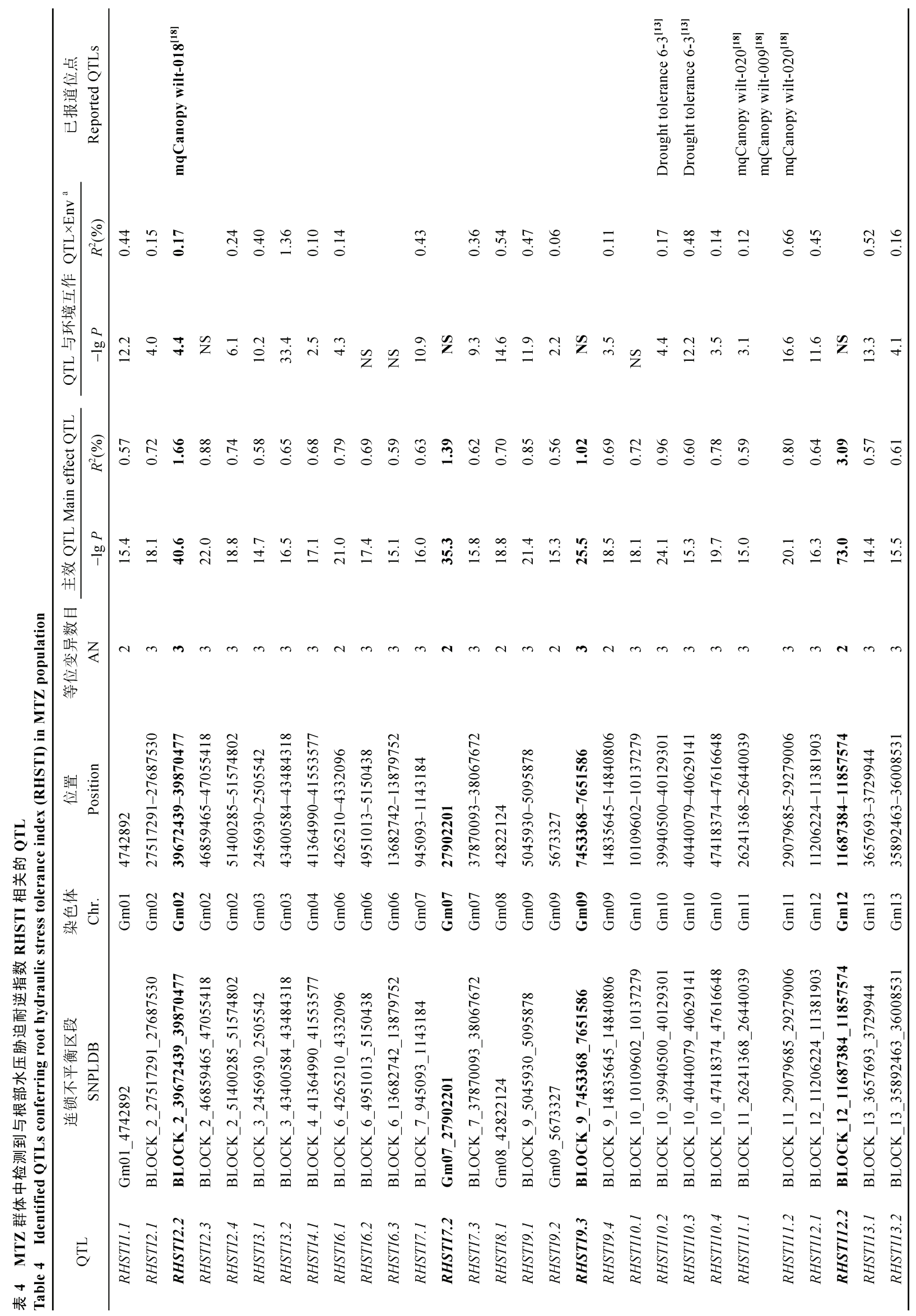




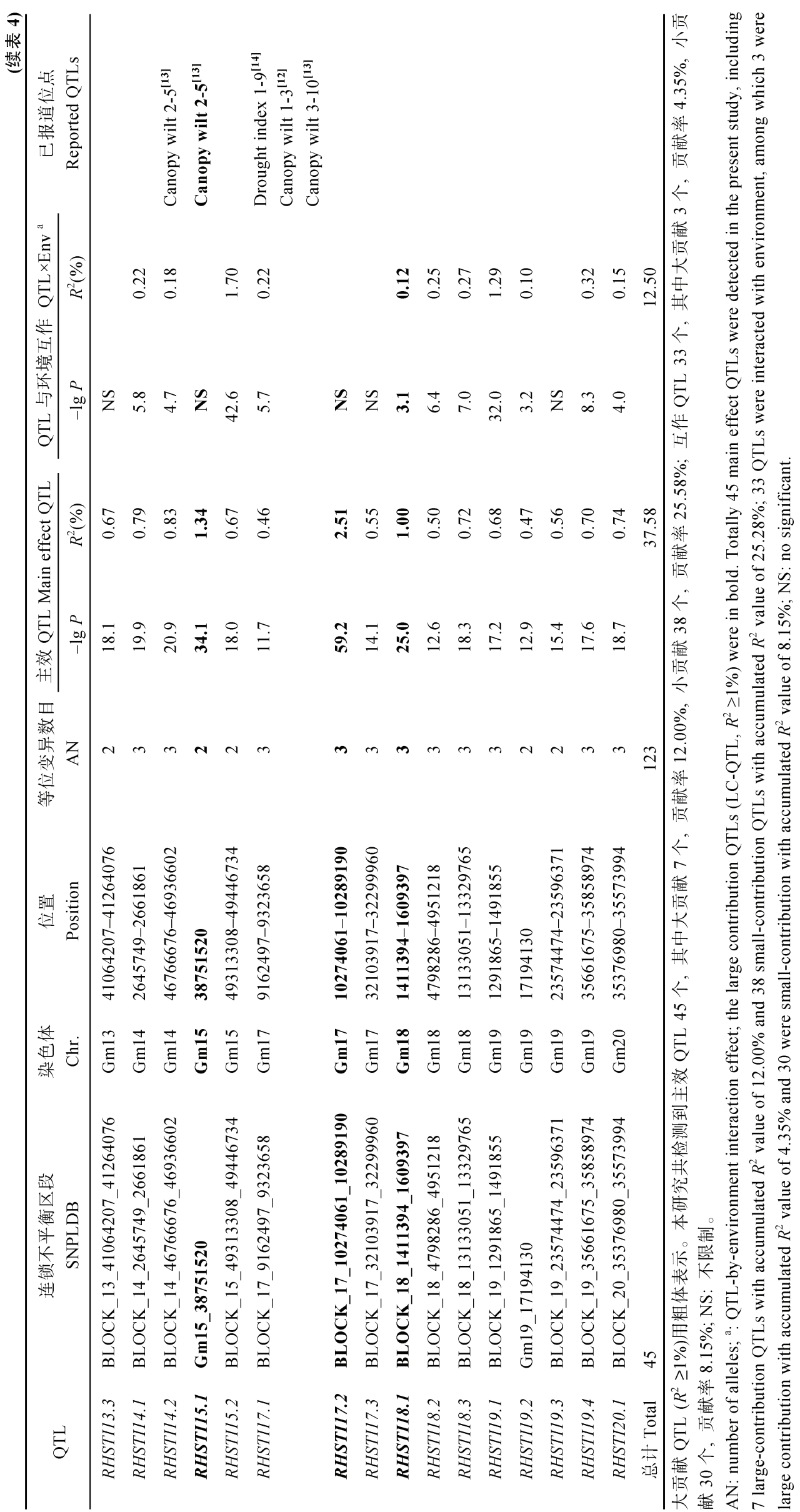



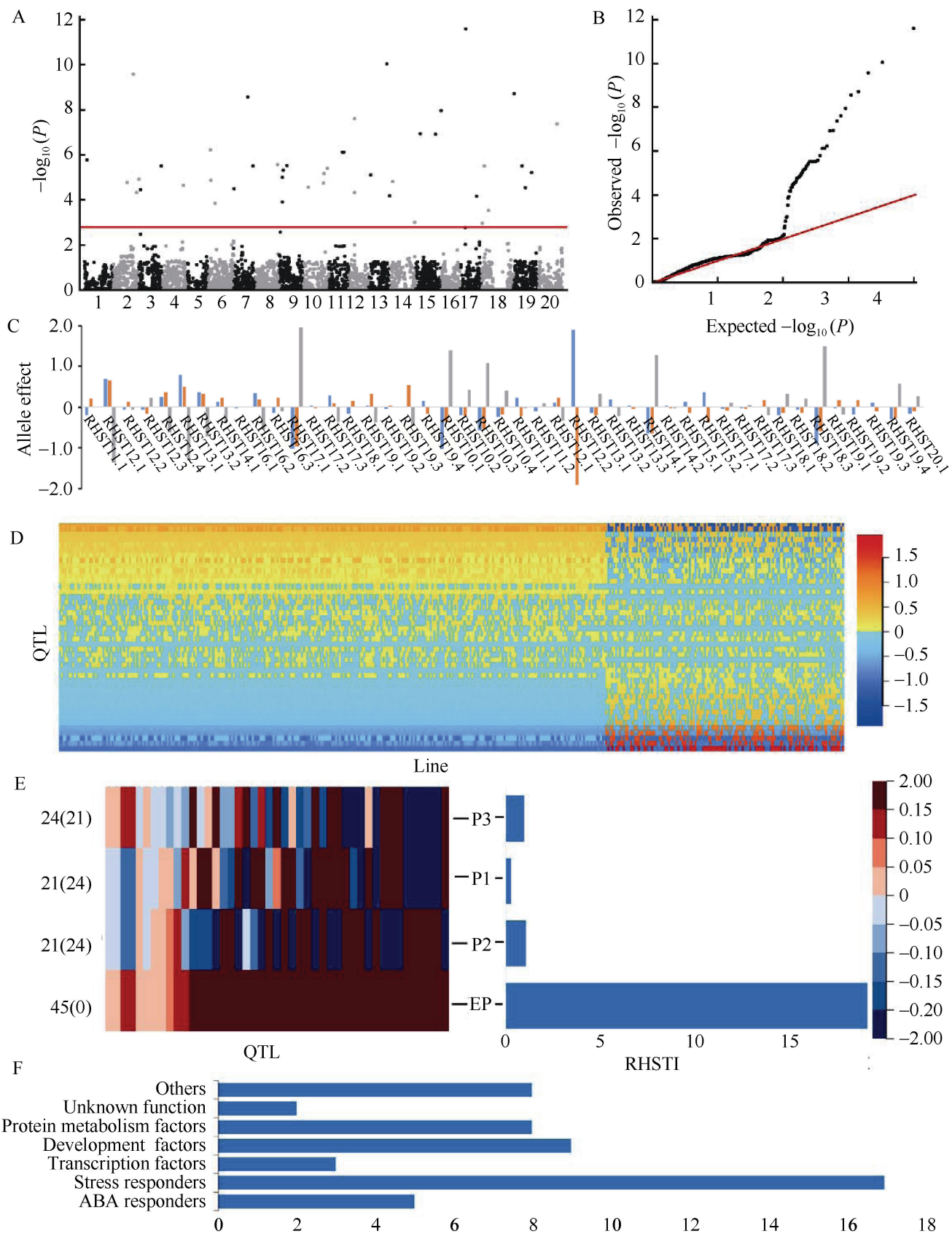

\section{图 2 MTZ 群体根部水压胁迫耐逆指数的遗传解析}

Fig. 2 Genetic analysis of RHSTI in the MTZ population

A: MTZ 群体中根部水压胁迫耐逆指数全基因组关联分析曼哈顿图。B: MTZ 群体中根部水压胁迫耐逆指数全基因组关联分析 QQ 图。 C: 123 个 RHSTI QTL 等位变异效应; 横坐标上方的条柱代表增效等位变异效应，横坐标下方的条柱代表减效等位变异效应。D: MTZ 群体 RHSTI QTL-allele 矩阵, 横轴为家系, 按根部水压胁迫耐逆指数由左向右递增排列, 竖轴为 QTL, 暖色表示增效等位基因, 冷色 表示减效等位基因, 颜色深度表示等位基因效应的大小。E: 3 个亲本和最优后代的表型及其遗传结构; 括号内外的数字分别代表材料 携带减效与增效等位变异数目; P1、P2、P3 和 EP 分别代表 3 个亲本蒙 8206、正阳白毛平顶、通山薄皮黄豆甲与聚合所有最优等位变 异的后代。F：RHSTI 候选基因 GO 功能聚类分析。

A: Manhattan plot of GWAS for RHSTI in MTZ population. B: Quantile-quantile plot of GWAS for RHSTI in MTZ population. C: the effects of 123 alleles on 45 RHSTI loci; the bars above the horizontal axis represent positive values while the bars below the horizontal axis represent negative values. D: the QTL-allele matrix of RHSTI in the MTZ population; the horizontal axis represents the lines of MTZ in ascending order according to RHSTI from the left side to the right side; the vertical axis is the QTL of RHSTI for each line and each horizontal indicates one allele with its effects expressed as the color thickness; the cells with warm colors indicate positive alleles; while the cells with cool colors indicate negative alleles the depth of the color indicates the size of the allele effect; E: the genetic structure and phenotype value of the three parents along with the elite progeny; the numbers in and outside of the parentheses represent the total numbers of negative and positive alleles, respectively; P1, P2, P3, and EP represent M8206, Zhengyangbaimaopingding, Tongshanbopihuangdoujia, and elite progeny pyramiding all the most favorable alleles, respectively. F: GO functional classification of the candidate genes of RHSTI. 
异中，等位变异效应变幅为-1.911 1.961; 其中, 58 个为增效等位变异, 变幅为 $0.001 \sim 1.961,65$ 个为减 效等位变异, 变幅为-1.911 -0.001 (图 2-C)。根据 上述结果, 本研究建立了 MTZ 群体 $45 \times 409$ 的 QTL-allele 矩阵, 横坐标代表 409 份半同胞家系, 纵 坐标表示 45 个 QTL位点, 每个位点上的等位变异用 其效应值表示, 颜色深浅表示等位变异效应大小(图 2-D)。随着家系根部水压胁迫耐逆指数的升高, 家系 携带增效等位变异有升高的趋势, 并且每个家系均 携带有增效的等位变异和减效等位变异, 表明家系 间具有较高的重组潜力。

根据遗传解析结果, 在本研究检测到的 45 个主 效位点上, 3 个亲本通山薄皮黄豆甲、蒙 8206 和正 阳白毛平顶分别携带有 24、21 和 21 个增效等位变 异, 另外 21、24 和 24 个为减效等位变异, 其 RHSTI 值分别为 $1.05 、 0.26$ 和 0.95 。不考虑互作, 只考虑 基因加性效应的情况下, 3 个亲本的最优重组个体即 在 45 个位点上均携带最优等位变异, 其 RHSTI 值可
以高达 18.92，显示了巨大的超亲潜力(图 2-E)。

2.4 大豆根部水压胁迫耐逆指数相关候选基因 分析

联合大豆苗期 PEG 胁迫转录组数据, 在根部水 压胁迫耐逆指数相关位点及其附近 $500 \mathrm{~kb}$ 区间内共 篮选到 38 个差异表达基因, 其中 24 个基因上调表 达, 相对表达量变幅 2.02 4,002,450.00 倍; 14 个基 因下调表达, 相对表达量变幅 $0.04 \sim 0.50$ 。位于 RHSTI9.1 位点上的候选基因 Glyma.09G051700 在 PEG 处理前后表达量差异高达 4,002,450.00 倍, 其 编码一个未知功能的蛋白。根据生物学功能, 38 个 耐旱候选基因可归为 7 类, 包括 ABA 响应因子、逆 境响应因子、转录因子、发育因子、蛋白代谢因子、 未知功能和其他, 分别包含 $5 、 17 、 3 、 9 、 8 、 2$ 和 8 个候选基因, 其中逆境响应因子和转录因子和发育 因子是最大的 3 类。表明, 大豆耐旱性是一个复杂 性状，有多个具有不同功能的候选基因共同控制。 具体结果见表 5 。

表 5 转录组与 GWAS 相结合的根部水压胁迫耐逆指数 RHSTI 候选基因分析

Table 5 Analysis the candidate genes of root hydraulic stress tolerance index (RHSTI) combined the results of GWAS and RNA-seq

\begin{tabular}{|c|c|c|c|c|c|c|c|c|c|c|}
\hline $\begin{array}{l}\text { 基因 } \\
\text { Gene }\end{array}$ & QTL & $\begin{array}{c}\text { 对照 } \\
\text { Control }\end{array}$ & $\begin{array}{c}\text { 处理 } \\
\text { Treatment }\end{array}$ & A & $\mathrm{S}$ & $\mathrm{T}$ & $\mathrm{D}$ & PM & UF & $\mathrm{O}$ \\
\hline Glyma.01G044100 & RHSTI1.1 & 4.63315 & 26.99810 & & & & & $\sqrt{ }$ & & \\
\hline Glyma.02G171100 & RHSTI2.1 & 1.65710 & 35.83620 & & $\sqrt{ }$ & & & & & \\
\hline Glyma.02G196500 & RHSTI2.2 & 13.39870 & 54.69290 & $\sqrt{ }$ & $\sqrt{ }$ & $\sqrt{ }$ & $\sqrt{ }$ & & & \\
\hline Glyma.02G250200 & RHSTI2.3 & 10.60250 & 154.59800 & $\sqrt{ }$ & $\sqrt{ }$ & & & $\sqrt{ }$ & & \\
\hline Glyma.02G308100 & RHSTI2.4 & 20.68510 & 89.04400 & & & & & & & $\sqrt{ }$ \\
\hline Glyma.03G023900 & RHSTI3.1 & 10.74360 & 39.75440 & & & & & & $\sqrt{ }$ & \\
\hline Glyma.03G202400 & RHSTI3.2 & 46.58860 & 2.89863 & & & & & $\sqrt{ }$ & & \\
\hline Glyma.04G179500 & RHSTI4.1 & 615.19900 & 78.03500 & $\sqrt{ }$ & $\sqrt{ }$ & & & $\sqrt{ }$ & & \\
\hline Glyma.06G054700 & RHSTI6.1 & 0.27378 & 132.47900 & & $\sqrt{ }$ & & & & & \\
\hline Glyma.06G063500 & RHSTI6.2 & 5.58627 & 26.31760 & & & & $\sqrt{ }$ & & & \\
\hline Glyma.06G165100 & RHSTI6.3 & 16.17140 & 3.43912 & & & & $\sqrt{ }$ & & & \\
\hline Glyma.07G013900 & RHSTI7.1 & 119.39400 & 5.21799 & & & & & $\sqrt{ }$ & & \\
\hline Glyma.07G208700 & RHSTI7.3 & 59.19030 & 196.58300 & & $\sqrt{ }$ & & $\sqrt{ }$ & & & \\
\hline Glyma.08G316700 & RHSTI8.1 & 8.69897 & 187.91100 & & & & & & & $\sqrt{ }$ \\
\hline Glyma.09G051700 & RHSTI9.1 & 0.00001 & 40.02450 & & & & & & $\sqrt{ }$ & \\
\hline Glyma.09G071600 & RHSTI9.3 & 5.55258 & 142.93600 & $\sqrt{ }$ & $\sqrt{ }$ & & $\sqrt{ }$ & & & \\
\hline Glyma.10G081700 & RHSTI10.1 & 18.56970 & 69.58230 & $\sqrt{ }$ & $\sqrt{ }$ & $\sqrt{ }$ & $\sqrt{ }$ & & & \\
\hline Glyma.10G169300 & RHSTI10.2 & 3.97157 & 66.91020 & & & & & $\sqrt{ }$ & & \\
\hline Glyma.10G179200 & RHSTI10.3 & 32.64080 & 6.27830 & & & & & & & $\sqrt{ }$ \\
\hline Glyma.10G251900 & RHSTI10.4 & 248.40400 & 501.60600 & & & & & $\sqrt{ }$ & & \\
\hline Glyma.11G171400 & RHSTI11.1 & 8.60429 & 102.58700 & & $\sqrt{ }$ & & & & & \\
\hline Glyma.11G207000 & RHSTI11.2 & 1.99196 & 20.33400 & & $\sqrt{ }$ & & & & & \\
\hline Glyma.12G112100 & RHSTI12.1 & 64.63140 & 29.13040 & & & & & & & $\sqrt{ }$ \\
\hline
\end{tabular}


(续表 5)

\begin{tabular}{|c|c|c|c|c|c|c|c|c|c|c|}
\hline $\begin{array}{l}\text { 基因 } \\
\text { Gene }\end{array}$ & QTL & $\begin{array}{c}\text { 对照 } \\
\text { Control }\end{array}$ & $\begin{array}{c}\text { 处理 } \\
\text { Treatment }\end{array}$ & A & $\mathrm{S}$ & $\mathrm{T}$ & $\mathrm{D}$ & $\mathrm{PM}$ & UF & $\mathrm{O}$ \\
\hline Glyma.12G117000 & RHSTI12.2 & 3.83243 & 33.14560 & & $\sqrt{ }$ & & & & & \\
\hline Glyma.13G078400 & RHSTI13.1 & 49.82870 & 14.74430 & & & $\sqrt{ }$ & & & & \\
\hline Glyma.13G272000 & RHSTI13.2 & 56.77120 & 135.84100 & & $\sqrt{ }$ & & & & & \\
\hline Glyma.13G333100 & RHSTI13.3 & 44.62830 & 847.65000 & & $\sqrt{ }$ & & & & & \\
\hline Glyma.14G190800 & RHSTI14.2 & 36.08840 & 9.31428 & & $\sqrt{ }$ & & & & & \\
\hline Glyma.15G222200 & RHSTI15.1 & 19.02410 & 1.79038 & & $\sqrt{ }$ & & & & & \\
\hline Glyma.15G267900 & RHSTI15.2 & 2.69447 & 34.25220 & & & & & & & $\sqrt{ }$ \\
\hline Glyma.17G113100 & RHSTI17.1 & 79.26370 & 26.09940 & & & & $\sqrt{ }$ & & & \\
\hline Glyma.17G122900 & RHSTI17.2 & 46.38730 & 23.03860 & & & & & & & $\sqrt{ }$ \\
\hline Glyma.17G202600 & RHSTI17.3 & 0.73104 & 40.50930 & & & & & & & $\sqrt{ }$ \\
\hline Glyma.18G018600 & RHSTI18.1 & 9.17402 & 136.37000 & & $\sqrt{ }$ & & $\sqrt{ }$ & & & \\
\hline Glyma.19G014800 & RHSTI19.1 & 31.60140 & 7.32146 & & $\sqrt{ }$ & & & & & \\
\hline Glyma.19G100600 & RHSTI19.3 & 53.01000 & 13.26570 & & & & & & & $\sqrt{ }$ \\
\hline Glyma.19G109400 & RHSTI19.4 & 25.26790 & 129.41800 & & & & & $\sqrt{ }$ & & \\
\hline Glyma.19G112700 & RHSTI20.1 & 119.13700 & 37.92940 & & & & $\sqrt{ }$ & & & \\
\hline
\end{tabular}

位于大贡献位点中的候选基因用粗体显示。对照和处理列的数字代表基因的 FPKM 值, 其表示定位到基因的片段数除以定位到基因 组的所有读长数(以 Million 为单位)与 RNA 的长度 (以 $\mathrm{kb}$ 为单位)。 $A 、 S 、 T 、 D 、 P M 、 U$ 和 $\mathrm{O}$ 分别代表 $\mathrm{ABA}$ 响应因子、逆境响应因 子、转录因子、发育因子、蛋白代谢因子、未知功能和其他等 7 类基因功能。

The gene in a large contribution QTL is in bold. In the column of control and treatments, the value is the gene's FPKM, which represents fragments per kilobase per million. A, S, T, D, PM, U, and O represent ABA responders, stress responders, transcription factors, development factors, protein metabolism factors, unknown function, and others, respectively.

\section{3 讨论}

3.1 大豆耐旱性遗传基础复杂，不同指标、不同 群体具有不同但相似的遗传体系

植物耐旱性评价主要有生理生化指标和生长形 态指标两大类。生理生化指标包括脯氨酸含量、丙 二醛含量、过氧化物气化酶含量和电导率等, 其是 植物受到外界胁迫时的直接反应, 因表型调查困难, 群体较大时较难进行; 生长指标较生理指标稳定, 表型鉴定成本低, 是植物耐旱性的最终反映。因而, 植物耐旱性是复杂的数量性状, 仅凭单个指标很难 对其进行综合评价。脯氨酸含量作为植物抗逆生理 生化研究中的一项重要指标, 已被用于水稻 ${ }^{[29]}$ 、小 麦 ${ }^{[30]}$ 、玉米 ${ }^{[31]}$ 等作物耐逆 QTL 定位研究中, 然而在 大豆中文献报道较少。

为解析大豆耐旱性的遗传基础, 本研究前期构 建了由 2 套重组自交系群体组成的 NAM 群体 MTZ, 并以生长指标评价了其耐旱性, 检测到 157 个耐旱 性 QTL ${ }^{[19-20]}$ 。本研究将耐旱性指标拓展到生理指标 脯氨酸含量, 评价了 MTZ 群体根部水压胁迫耐逆指 数, 检测到 45 个 QTL 位点, 包含 123 个等位变异, 这些位点包括 7 个大贡献 QTL 和 38 个小贡献 QTL,
累计解释 37.58\%的表型变异。同时发现大部分位点 与环境存在互作, 互作可以解释 $12.50 \%$ 的表型变 异。与 Mueen 等 ${ }^{[19-20]}$ 利用 MTZ 群体检测到的相对 根长、相对茎长、相对植株长度(根+茎)和相对干重 等生长指标耐旱性 QTL 结果相整合, 共检测到 18 个 QTL 簇与多个指标相关, 分布在大豆 3 号、4 号、 6 号、7 号、10 号、13 号、14 号、15 号、17 号、 18 号、19 号和 20 号染色体上, 其中有 3 个 QTL 簇 所包含的位点均为大贡献 QTL, 具体见表 6 。这些 QTL 簇, 尤其与多个性状相关联的 QTL 簇应为控制 大豆耐旱性的主要染色体区段。上述结果说明, 大 豆耐旱性是一个复杂性状, 不同指标具有不同的遗 传体系，共有位点仅占较小部分 $[16 /(45+157)]$ 。

与前人检测到的耐旱性 QTL 相比, 本研究检测 到的 45 个 QTL 位点中, 有 8 个位点与前人耐旱性位 点定位结果一致(表 4)。RHSTI10.2 和 RHSTI10.3 已 被前人在“Benning/PI416937"[13]群体中已检测到; RHSTI14.2 和 RHSTI15.1 位点已被前人在“Benning/ PI416937” "[13]群体中以叶片萎蒸程度为指标检测到。 RHSTI17.1 已被前人在“科丰 1 号/南农 1138-2”[14]、 “KS4895/Jackson”[12]和“93705 KS4895/Jackson”[13] 等群体中检测到, 另外 3 个位点 RHSTI2.2、 
表 6 多个耐旱指标评价 MTZ 群体检测到的 QTL 簇 $( \pm \mathbf{5 0 0} \mathbf{~ k b})$

Table 6 QTL clusters of multiple drought tolerance indicators within $500 \mathrm{~kb}$ in MTZ population

\begin{tabular}{|c|c|c|c|c|c|c|c|}
\hline $\begin{array}{l}\text { 序号 } \\
\text { Order }\end{array}$ & QTL & $\begin{array}{l}\text { 染色体 } \\
\text { Chr. }\end{array}$ & $\begin{array}{c}\text { 物理位置 } \\
\text { Physical position }\end{array}$ & $\begin{array}{l}\text { 序号 } \\
\text { Order }\end{array}$ & QTL & $\begin{array}{c}\text { 染色体 } \\
\text { Chr. }\end{array}$ & $\begin{array}{c}\text { 物理位置 } \\
\text { Physical position }\end{array}$ \\
\hline 1 & RHSTI3.2/RRL3.5 & $\mathrm{Gm} 03$ & $43400584-43484318$ & 10 & RRL14.1/RPDW14.2 & Gm14 & $47717307-48064975$ \\
\hline 2 & RSL4.2/RPL4.1 & $\mathrm{Gm} 04$ & $44742587-45097286$ & 11 & RRL15.1/RSL15.1 & Gm15 & $11105948-11421179$ \\
\hline 3 & RHSTI6.3/RRL6.2 & Gm06 & $13682742-13879752$ & 12 & RHSTI15.2/RRL15.4 & Gm15 & 48985599-49446734 \\
\hline 4 & RHSTI7.3/RRL7.2 & $\mathrm{Gm} 07$ & $37870093-38067672$ & 13 & RHSTI17.3/RRL17.2 & $\mathrm{Gm} 17$ & $32103917-34132465$ \\
\hline 5 & RHSTI10.3/RRL10.2 & Gm10 & $40440079-40629141$ & 14 & RHSTI18.1/RPL18.1 & $\mathrm{Gm} 18$ & 1411394-2099117 \\
\hline 6 & $R R L 10.3 / \boldsymbol{R P D W 1 0 . 1}$ & Gm10 & $50470068-50478746$ & 15 & RHSTI19.2/RSL19.3 & Gm19 & $16552234-17194130$ \\
\hline 7 & RPL13.2/RPDW13.1 & $\mathrm{Gm} 13$ & $22001544-22395896$ & 16 & RHSTI19.4/RRL19.3 & Gm19 & $35661675-35924257$ \\
\hline 8 & RHSTI13.2/RSL13.1 & Gm 13 & $35347991-36008531$ & 17 & RRL19.5/RPDW19.1 & Gm19 & $37678898-38456131$ \\
\hline 9 & RRL13.6/RPL13.1 & $\mathrm{Gm} 13$ & $43533843-43640816$ & 18 & RHSTI20.1/RPL20.1 & Gm20 & 35319103-35573994 \\
\hline
\end{tabular}

$R S L 、 R R L$ 和 $R P D W$ 表示 Mueen 等 ${ }^{[19-20]}$ 以相对茎长、相对根长和相对干重为指标在 MTZ 群体中检测到的 QTL 位点, 大贡献位点 $\left(R^{2} \geq\right.$ $1 \%)$ 用粗体表示。

$R S L, R R L$, and $R P D W$ represent the QTLs associated with relative shoot length, relative root length and relative plant weight detected in MTZ population by Mueen et al. ${ }^{[19-20]}$, and the QTLs with $R^{2} \geq 1 \%$ are shown in bold.

RHSTI11.1 和 RHSTI11.2 已被前人在多个群体 QTL 元分析 ${ }^{[18]}$ 中检测到。表明，大豆耐旱性是一个复杂 数量性状，遗传基础复杂，不同的群体具有不能的 遗传基础; 能被多个群体检测到的位点仅占少数, 应为控制大豆耐旱性最为主要的 QTL。

\section{2 大豆耐旱性涉及一套具有不同功能的候选} 基因体系

借助高密度的分子标记, 本研究可以将根部水 压胁迫耐逆指数位点定位到较小的染色体区段内, 便于候选基因的预测。结合 PEG 胁迫下转录组数据, 共预测到 38 个耐旱性候选基因, 可归为 7 类, 包括 ABA 响应因子、逆境响应因子、转录因子、发育因 子、蛋白代谢、未知功能和其他, 其中逆境响应因 子、转录因子、发育因子是最大的 3 类。3 8 个根部 水压胁迫耐逆指数候选基因中，有 6 个基因位于大 贡献位点上, 其中 RHSTI2.2 位点上的候选基因 Glyma.02G196500 属于 BLH1 类转录因子, 参与 $\mathrm{ABA}$ 刺激、耐盐和生长发育等多个生物学过程; RHSTI9.3 位点上的候选基因 Glyma.09G071600 编码 中茉莉酸信号蛋白, 参与了 $\mathrm{ABA}$ 信号、缺水响应和 花的发育等 27 个生物学过程; RHSTI11.2 位点上的 候选基因 Glyma.11G207000 参与应对高温和蛋白磷 酸化等生物学过程; RHSTI15.1 位点上的候选基因 Glyma.15G222200 参与了植物应对缺水的生物学过 程；RHSTI18.1 位点上的候选基因 Glyma. $18 G 018600$ 参与了应对高温和低磷等生物学过程。 表明，大豆耐旱性是一个复杂性状，有多个具有不 同功能的候选基因共同控制，位于大贡献位点上的
候选基因应为控制大豆耐旱性的主要基因，具有深 入研究价值。

\section{4 结论}

PEG 模拟干旱条件下, MTZ 群体及其亲本在根 部水压胁迫耐逆指数上存在显著差异, 且群体表型 变异丰富。根部水压胁迫耐逆指数在单个环境下具 有较高遗传力，在不同环境间存在较强互作，说明 MTZ 群体根部水压胁迫耐逆指数受遗传和环境的共 同影响。利用限制性二阶段多位点全基因组关联分 析方法，对 MTZ 群体遗传解析发现，根部水压胁迫 耐逆指数遗传基础复杂, 由小贡献和大贡献的位点 共同控制，同时位点与环境间存在显著互作。对定 位区间的候选基因进行注释，共预测到 38 个候选基 因, 涉及 $\mathrm{ABA}$ 响应因子、逆境响应因子、转录因子、 发育因子、蛋白代谢、未知功能和其他等不同功能, 其中逆境响应因子、转录因子、发育因子是最大的 3 类, 位于大贡献位点的 6 个候选基因参与 $\mathrm{ABA}$ 、 逆境和生长发育等多个生物学过程, 具有重要研究 价值。上述研究结果表明大豆耐旱性是一个由多位 点、多基因控制的复杂数量性状, 且与环境存在互 作，遗传基础复杂。

\section{References}

[1] Reynolds J F, Smith D M S, Lambin E F, Turner B L, Mortimore M, Batterbury S P J, Downing T E, Dowlatabadi H, Fernández R J, Herrick J E, Huber-Sannwald E, Jiang H, Leemans R, Lynam T, Maestre F T, Ayarza M, Walker B. Global desertification: Building a science for dryland development. Science, 2007, 316: 847-851. 
[2] Cook E R, Seager R, Cane M A, Stahle D W. North American drought: reconstructions, causes, and consequences. Earth Sci Rev, 2007, 81: 93-134.

[3] Frederick J R, Camp C R, Bauer P J. Drought-stress effects on branch and mainstem seed yield and yield components of determinate soybean. Crop Sci, 2001, 41: 759-763.

[4] Sadeghipour O, Abbasi S. Soybean response to drought and seed inoculation. World Appl Sci J, 2012, 17: 55-60.

[5] Sloane R J, Patterson R P, Carter T E. Field drought tolerance of a soybean plant introduction. Crop Sci, 1990, 30: 118-123.

[6] 刘荣, 盖钧镒, 吕慧能, 王永军, 陈受宜. 大豆耐旱种质鉴定 和相关根系性状的遗传与 QTL 定位. 遗传学报, 2005, 32: 855-863.

Liu Y, Gai J Y, Lyu H N, Wang Y J, Chen S Y. Identification of drought tolerant germplasm and inheritance and QTL mapping of related root traits in soybean (Glycine $\max$ (L.) Merr.). Acta Genet Sin, 2005, 32: 855-863 (in Chinese with English abstract).

[7] Liu F, Andersen M N, Jacobsen S E, Jensen C R. Stomatal control and water use efficiency of soybean (Glycine max L. Merr.) during progressive soil drying. Environ Exp Bot, 2005, 54: 33-40.

[8] Guo Y, Yu H, Yang M, Kong D, Zhang Y. Effect of drought stress on lipid peroxidation, osmotic adjustment and antioxidant enzyme activity of leaves and roots of Lycium ruthenicum Murr. seedling. Russ J Plant Physiol, 2018, 65: 244-250.

[9] 耳阝兴元, 何晖, 张燕, 朱献辉. 脯氨酸对高温胁迫下猕猴桃苗 抗热性相关生理指标的影响. 山东农业科学, 2010, (5): 44-46. Ye X Y, He H, Zhang Y, Zhu X H. Effects of proline on physiological indexes related to heat resistance of kiwifruit seedlings under high temperature stress. Shandong Agric Sci, 2010, (5): 44-46 (in Chinese with English abstract).

[10] 王涛, 田雪瑶, 谢寅峰, 张往祥. 植物耐热性研究进展. 云南 农业大学学报(自然科学), 2013, 28: 719-726.

Wang T, Tian X Y, Xie Y F, Zhang W X. Research advance on heat-stress tolerance in plants. J Yunnan Agric Univ (Nat Sci), 2013, 28: 719-726 (in Chinese with English abstract).

[11] Abdel-Haleem H, Carter T E, Purcell L C, King C A, Ries L L, Chen P Y, Schapaugh W, Sinclair T R, Boerma H R Mapping of quantitative trait loci for canopy-wilting trait in soybean (Glycine max L. Merr.). Theor Appl Genet, 2012, 125: 837-846.

[12] Charlson D V, Bhatnagar S, King C A, Ray J D, Sneller C H, Carter T E, Purcell L C. Polygenic inheritance of canopy wilting in soybean [Glycine max (L.) Merr.]. Theor Appl Genet, 2009, 119: 587-594.

[13] Hwang S D, King C A, Ray J D, Cregan P B, Chen P, Carter T E, Li Z L, Abdel-Haleem H, Matson K W, Schapaugh W, Purcell L C. Confirmation of delayed canopy wilting QTLs from multiple soybean mapping populations. Theor Appl Genet, 2015, 128: 2047-2065.

[14] Du W J, Wang M, Fu S X, Yu D Y. Mapping QTLs for seed yield and drought susceptibility index in soybean (Glycine $\max$ L.) across different environments. J Genet Genomics, 2009, 36: 721-731.

[15] Carpentieri-Pipolo V, Pipolo A E, Abdel-Haleem H, Boerma H R, Sinclair T R. Identification of QTLs associated with limited leaf hydraulic conductance in soybean. Euphytica, 2012, 186: 679-686.
[16] Mian M A R, Ashley D A, Boerma H R. An additional QTL for water use efficiency in soybean. Crop Sci, 1998, 38: 390-393.

[17] Mian M A R, Bailey M A, Ashley D A, Wells R, Carter T E, Parrott W A, Boerma H R. Molecular markers associated with water use efficiency and leaf ash in soybean. Crop Sci, 1996, 36: 1252-1257.

[18] Hwang S, King C A, Chen P Y, Ray J D, Cregan P B, Carter T E, Li Z L, Abdel-Haleem H, Matson K W, Schapaugh W, Purcell L C. Meta-analysis to refine map position and reduce confidence intervals for delayed-canopy-wilting QTLs in soybean. Mol Breed, 2016, 36.

[19] Khan M A, Tong F, Wang W B, He J B, Zhao T J, Gai J Y. Using the RTM-GWAS procedure to detect the drought tolerance QTL-allele system at the seedling stage under sand culture in a half-sib population of soybean [Glycine $\max ($ L.) Merr.]. Can J Plant Sci, 2019, 99: 801-814.

[20] Khan M A, Tong F, Wang W B, He J B, Zhao T J, Gai J Y. Analysis of QTL-allele system conferring drought tolerance at seedling stage in a nested association mapping population of soybean [Glycine $\max (\mathrm{L}$.) Merr.] using a novel GWAS procedure. Planta, 2019, 249: 1653-1653.

[21] 张志良, 翟伟菁. 植物生理学实验指导. 北京: 高等教育出版 社, 2003. pp 258-260.

Zhang Z L, Qu W J. Guide to Plant Physiology. Beijing: High Education Press, 2003. pp 258-260 (in Chinese).

[22] Sayed M A, Schumann H, Pillen K, Naz A A, Leon J. AB-QTL analysis reveals new alleles associated to proline accumulation and leaf wilting under drought stress conditions in barley (Hordeum vulgare L.). BMC Genetics, 2012, 13: 61.

[23] Andolfatto P, Davison D, Erezyilmaz D, Hu T T, Mast J, Sunayama-Morita T, Stern D L. Multiplexed shotgun genotyping for rapid and efficient genetic mapping. Genome Res, 2011, 21: 610-617.

[24] Li R Q, Yu C, Li Y R, Lam T W, Yiu S M, Kristiansen K, Wang J. SOAP2: an improved ultrafast tool for short read alignment. Bioinformatics, 2009, 25: 1966-1967.

[25] Yi X, Liang Y, Huerta-Sanchez E, Jin X, Cuo Z X P, Pool J E, Xu X, Jiang H, Vinckenbosch N, Korneliussen T S, Zheng H C, Liu T, He W M, Li K, Luo R B, Nie X F, Wu H L, Zhao M R, Cao H Z, Zou J, Shan Y, Li S Z, Yang Q, Asan, Ni P X, Tian G, Xu J M, Liu X A, Jiang T, Wu R H, Zhou G Y, Tang M F, Qin J J, Wang T, Feng S J, Li G H, Huasang, Luosang J B, Wang W, Chen F, Wang Y D, Zheng X G, Li Z, Bianba Z M, Yang G, Wang X P, Tang S H, Gao G Y, Chen Y, Luo Z, Gusang L, Cao Z, Zhang Q H, Ouyang W H, Ren X L, Liang H Q, Zheng H S, Huang Y B, Li J X, Bolund L, Kristiansen K, Li Y R, Zhang Y, Zhang X Q, Li R Q, Li S G, Yang H M, Nielsen R, Wang J, Wang J A. Sequencing of 50 human exomes reveals adaptation to high altitude. Science, 2010, 329: 75-78.

[26] Scheet P, Stephens M. A fast and flexible statistical model for large-scale population genotype data: applications to inferring missing genotypes and haplotypic phase. Am J Hum Genet, 2006, 78: 629-644.

[27] Barrett J, Fry B, Maller J, Daly M. Haploview: analysis and visualization of LD and haplotype maps. Bioinformatics, 2005, 21: 263-265. 
[28] He J, Meng S, Zhao T, Xing G, Yang S, Li Y, Guan R, Lu J, Wang Y, Xia Q, Yang B, Gai J. An innovative procedure of genome-wide association analysis fits studies on germplasm population and plant breeding. Theor Appl Genet, 2017, 130: 2327-2343.

[29] Barik S R, Pandit E, Mohanty S P, Nayak D K, Pradhan S K. Genetic mapping of physiological traits associated with terminal stage drought tolerance in rice. BMC Genetics, 2020, 21: 1-12.

[30] Ilyas N, Amjid M W, Saleem M A, Khan W, Wattoo F M, Rana R M, Maqsood, Rana H, Zahid A, Shah G A, Anwar A. Ahmad M Q,
Shaheen M, Riaz H, Ansari M J. Quantitative trait loci (QTL) mapping for physiological and biochemical attributes in a Pasban 90/Frontana recombinant inbred lines (RILs) population of wheat (Triticum aestivum) under salt stress condition. Saudi J Biol Sci, 2020, 27: 341-351.

[31] Zhang C, Jin F, Li S, Liu W, Ma X, Yang S, Li X. Fine mapping of major QTLs for alkaline tolerance at the seedling stage in maize (Zea mays L.) through genetic linkage analysis combined with high-throughput DNA sequencing. Euphytica, 2018, 214: 120. 